Scope, scale, and dose of the world's largest school based mental health programs

J. Michael Murphy, Ed.D. Department of Psychiatry, Massachusetts General Hospital, Boston MA Harvard Medical School, Boston, MA

Madelaine R. Abel, B.A. Clinical Child Psychology Program, University of Kansas, Lawrence, KS

Sharon Hoover Stephan, Ph.D. Department of Psychiatry, University of Maryland School of Medicine, Baltimore, MD

Michael Jellinek, M.D. Departments of Psychiatry and Pediatrics, Harvard Medical School, Boston, MA

Mina Fazel, D.M., MRC Psych Department of Psychiatry, Oxford University, Oxford, England Warneford Hospital, Oxford, England

Corresponding Author: J. Michael Murphy, EdD, Child Psychiatry Service, Massachusetts General Hospital, Yawkey 6A, Boston, MA 02114; [mmurphy6@partners.org]; Phone: (617) 510-3927; Fax: (617) 371-4802

Keywords: School-based interventions, children's mental health, large-scale programs 


\begin{abstract}
Untreated mental health problems are among the most disabling, persistent and costly of all health conditions, often beginning in childhood and persisting into adulthood. For these reasons, there has been growing interest in preventive mental health programs for children. In recent years, several such programs have been implemented at regional, state, or national scale and have now reached tens of millions of children. Although many experimental studies have documented positive outcomes of individual programs, this is the first attempt at a systematic comparison of the largest programs in terms of scope, scale, and dose.

The school-based mental health programs discussed in this review appear to have reached more than 27 million children over the last decade and many of these programs have collected systematic data on outcomes. The role that such programs can play in LMIC is a secondary focus of this paper. Until recently, widely-scaled preventive mental health interventions for children have been studied almost exclusively in HIC, despite the needs elsewhere, given that $80 \%$ of the global population of children reside in LMIC. Since a number of programs are now operating on a large scale in LMIC - as well as in HIC - it is now possible to consider child mental health programs from a global perspective. With both the increasing diversity of countries represented and the growing scale of programs, datasets of increasing quality and size are becoming available, opening up new opportunities to conduct the kinds of analyses that would be needed to assess the degree to which preventive interventions can be delivered at scale for child mental health problems and potentially play a role in improving health and other life outcomes.
\end{abstract}

Keywords: School based interventions; children’s mental health; large scale programs; secondary; prevention 


\section{Introduction (5766)}

The need to prioritize child and adolescent mental health is compelling since an estimated $13 \%$ of those under 18 years, worldwide, have significant mental health problems. ${ }^{1}$ The most common disorders are anxiety, disruptive behavior disorders, attention deficit hyperactivity disorder (ADHD), and depression. Numerous studies have shown that child mental health problems often persist into adulthood, especially if untreated in childhood, ${ }^{2}$ and that adult mental illness is among the most disabling and costly conditions in public health. ${ }^{3}$ The high prevalence of mental health problems and their associated negative outcomes, not only for long-term mental health but also with respect to educational and occupational attainment as well as impaired social relationships, suggest that finding ways to prevent mental health problems should be a major priority. ${ }^{4}$ For these reasons, there has been considerable interest in searching for effective and scalable interventions that could improve mental health from childhood to adulthood. Since the majority of children and adolescents spend considerable time in schools, school-based programs have received increasing attention., ${ }^{5,6}$ Although results from experimental studies have demonstrated significantly improved outcomes for children who participate in such programs, until now there has been little evidence to suggest that such programs could be implemented at scale in LMIC or HIC and even less evidence that, if implemented at scale, these programs would lead to population-wide improvements in mental health, health, educational, and/or social outcomes.

A recent editorial in the Journal of the American Academy of Child and Adolescent Psychiatry suggested that to address this gap, “large-scale programs need to be ...evaluated as they are created and implemented in real-world settings, and further refined as evidence is generated”. ${ }^{7}$ The current paper is consistent with this recommendation. Over the past few years, 
the scale and scope of children's mental health programs has increased considerably, now reaching whole districts, counties, states, provinces, and even whole countries with tens of millions of children involved. The establishment of large-scale programs in real world settings has also created new opportunities for cross program comparisons, continuous quality improvement, and possibly, soon, the evaluation of population scale improvements. Toward these ends, the current review attempted to identify the largest preventive interventions for children's mental health and to compare them in terms of their scope, scale, and dose.

The degree to which such programs have been implemented in LMIC is a secondary focus of this paper. Until recently, widely-scaled preventive mental health interventions for children have been studied almost exclusively in HIC, despite the fact that about $80 \%$ of the global population of children reside in LMIC. ${ }^{6}$ Since a number of programs are currently operating at a large scale in LMIC, it is now possible to consider child mental health programs from a global perspective. With both the growing scale of programs and the increasing diversity of countries represented, datasets of increasing quality and size are becoming available. The access to large datasets make it possible to conduct the analyses needed to assess the impact of preventive interventions for child mental health on improving population health in a wide range of real world settings.

\section{Method}

To identify the largest programs we conducted an initial search using PsycINFO and Ovid MEDLINE for articles published in English before December 2015 with combinations of the key search terms “school” or "school-based”, “mental health”, “therapy”, “treatment”, “program”, “intervention”, “prevention”, and “national” or “state”. This search identified 497 references. After the exclusion of duplicates and studies that did not examine children or mental 
health interventions, 45 studies remained. Several of the references identified were review articles and we examined the programs mentioned in those reviews for other possible program candidates. We also reviewed the reference lists of three recent review papers on preventive interventions in $\mathrm{HIC},{ }^{5} \mathrm{LMIC},{ }^{6}$ and social emotional learning programs ${ }^{8}$ to identify any additional large scale mental health programs, as well as contacting experts in the field. We focused this review on mental health preventive interventions for children implemented in school settings. Although programs focused on other types of interventions (such as those for substance abuse, anger management, or suicide prevention or those aimed at more global social-emotional learning) sometimes measure indicators like psychiatric impairment or societal outcomes (e.g., employment, disability, violence, or quality of life) which are of importance in public health, we excluded such programs unless they specifically mentioned the term 'mental health' in their overall program goals and measured specific mental health or illness variables in their program evaluations. The final result was a list of eight programs (see Table 1) that met all of these criteria. We then contacted a developer or key researcher for each of the programs and conducted a telephone interview with him/her in order to better understand specific program conceptualization and dissemination (see Appendix I, available online, for the contact person and website for each program).

Following a widely accepted health service categorization, we used the three-tiered model of interventions to compare programs. Figure 1 illustrates the model. Tier 1 programs focus on mental health promotion or primary prevention and are applied to whole populations (e.g., all of the students in a given school or classroom). Tier 2 interventions target specific vulnerable populations (e.g., children who have experienced potentially traumatic events and are already demonstrating some problems). Tier 3 interventions are aimed at supporting children 
with diagnosed disorders. This review focuses primarily on prevention and therefore on Tiers 1 and 2 (since Tier 3 is often synonymous with actual treatment). Several of the programs we review include Tier 3 components but they were not excluded although programs that focused exclusively on Tier 3 were.

It is important to make early note of the limitations inherent in studying this area, for while numerous child mental health preventive interventions have been created and implemented, they are still difficult to categorize precisely. Locations for intervention, target populations, and child characteristics selected for intervention include many possible sources of heterogeneity, as well as the unique qualities of each specific school setting. For these reasons this review should be viewed more as a heuristic process or an attempt to gain perspective during a time of innovation than as a definitive list of programs.

\section{School-Based Mental Health Programs Reviewed}

The eight school-based preventive mental health interventions identified as having reached the largest populations of children are Positive Behavior Interventions and Supports (PBIS); ${ }^{9}$ FRIENDS; ${ }^{10}$ Positive Action (PA); ${ }^{11}$ Promoting Alternative Thinking Strategies (PATHS); ${ }^{12}$ Skills for Life (SFL); ${ }^{13}$ MindMatters; ${ }^{14}$ the Good Behavior Game (GBG); ${ }^{15}$ and Cognitive-Behavioral Interventions for Trauma in Schools (CBITS). ${ }^{16}$ Seven of these were developed in HIC (either the USA or Australia) and the eighth (SFL) was developed in a LMIC (Chile). Each program is described below with particular emphasis on results of randomizedcontrol trials (RCTs) and program efficacy.

Positive Behavior Interventions and Supports (PBIS). PBIS, one of the most widely implemented programs reviewed in this paper, emphasizes a positive social culture and establishes sufficient behavioral support for all students through a three-tiered prevention model. 
Several studies provide evidence that PBIS promotes the successful management of school culture and children's academic achievement. ${ }^{17-19}$ Program effectiveness is particularly strong overtime (up to 5+ years) and when program is implemented with high fidelity. ${ }^{20,21}$ For example, across studies, academic achievement is a primary target (e.g., reading assessments and school outcomes such as suspensions) with effect sizes ranging from $1.08-1.71 .^{21}$ Notably, effects (particularly relating to school outcomes data) have been sustained consistently over long term follow-up periods (up to $5+$ years). ${ }^{22}$

FRIENDS. FRIENDS is a manualized cognitive-behavioral based program aimed at reducing anxiety and other internalizing disorders which teaches children, parents, and teachers skills for managing emotions and coping with stress in a resilient/positive manner. There are six primary topics: (1) identifying feelings; (2) understanding one’s physiological response; (3) learning to relax; (4) linking thoughts and feelings; (5) developing plans for coping; and (6) practicing emotion management. Several RCTs have been conducted to date and results across trials demonstrate moderate to large effect sizes at baseline (Cohen’s d = .22 - .44), and sustainability of effects at 6- and 12-month follow-up periods. ${ }^{23-25}$ Parent training components provide additional support above and beyond in-school program exposure, ${ }^{26}$ particularly among nonclinical (subclinical threshold) populations of youth with anxiety. Method of delivery has also been assessed and program implementation by health personnel (e.g., psychologists) appears to have greater effects on reduction of mental health outcomes than those delivered by school/education professionals. ${ }^{25}$ Overall, the FRIENDS program is designed as an anxiety preventive intervention and thus reductions in anxiety, as demonstrated across multiple studies, are consistent with the program's theoretical model. 
Positive Action (PA). Positive Action targets social emotional learning from PreK-12 grade students. Lessons focus on self-management skills, social skills, character building, and mental health. There are six primary units that encourage 'positive actions': (1) actions for improving the self; (2) being honest with the self and others; (3) getting along with others; (4) managing the self; (5) body and mind; and (6) philosophy and the thoughts-actions-feelings about self. One matched-pair randomized control trial demonstrated a 37\% decrease in violencerelated behaviors, a 27\% decrease in disruptive behaviors, and a 41\% decrease in bullying among students who participated in the program vs. a control group. ${ }^{27}$ Similar effects (specifically, doseresponse effects) have occurred long-term, particular among disciplinary behavior reductions and academic achievement improvements. ${ }^{28,29}$

Promoting Alternative Thinking Strategies (PATHS). The PATHS curriculum targets 5 major domains of functioning, including self-control, emotional understanding, positive selfesteem, relationships, and interpersonal problem-solving skills. An additional goal is the reduction of aggression and other problem behaviors. Lesson activities are comprised of roleplaying, story-telling, and attribution training. According to the CASEL Social-Emotional Learning (SEL) program guide, ${ }^{30}$ PATHS incorporates several factors essential for effective, school-based SEL implementation including integration of approach and theory, multi-grade level design, focus on the role of emotions and emotional development, ongoing support and training, and multiple outcome measures for program assessment. RCT research on PATHS generally suggests that if implemented with fidelity, PATHS practices are associated with reduced disciplinary infractions and aggression in school in the US but these findings have not been replicated in a UK study. Of note, several cluster randomized control studies have demonstrated that control students have increases in normative beliefs about aggression over 
time, as compared to the PATHS students’ improvements in non-violent interpersonal functioning (e.g., non-violent problem solving and non-aggressive social skills). ${ }^{31,32}$ Effects on academic achievement has also been documented; writing proficiency and math proficiency were 1.5 and 1.63 times higher, respectively, for intervention vs. control group. ${ }^{33}$ While these effects are relatively small for academic achievement, this is expected given that academic improvements are not the primary target of PATHS (conduct behaviors are). The fact that effects have carried over into academic achievement suggests the broader influence of PATHS on multiple areas of child well-being.

Skills for Life (SFL). The Skills for Life intervention is based on the three-tiered model recommended by the World Health Organization. Although the program was developed by trained clinicians and founded based on a national mental health standard, the program workshops and overall approach is skill-based. Results from a large national quasi-experimental study ${ }^{34}$ provided preliminary evidence for the impact of participation in the Tier 2 intervention on a number of behavioral and academic outcomes was significant. At risk students who attended more sessions of the intervention showed greater improvements in parent- and teacherrated behavioral health measure scores and in annual school attendance than did at risk students who attended fewer sessions. Effect sizes ranged from .08-.16 and which, while small, were deemed to be important given the two year follow up interval from first through third grades.

MindMatters. MindMatters is a mental health promotion program that embeds practices into the every-day school curriculum with a particular focus on promoting mental health awareness (coping skills, bullying, resiliency). ${ }^{14,35}$ Specific techniques include social and emotional learning programs, emotion regulation strategies, and coping skills for stress and 
anxiety. ${ }^{14}$ While no RCTs have been completed to date, several quasi-experimental studies have demonstrated its effectiveness in improving behavioral and academic outcomes.

Good Behavior Game (GBG). The Good Behavior Game is a universal classroom prevention program for high risk students that targets disruptive classroom behaviors, predominantly for primary school-aged children. It is a game played within the classroom setting; goals include the promotion of self-regulation and teamwork. ${ }^{15}$ Most notably, long-term outcomes from childhood through young adulthood demonstrate the significant influence of the game on minimizing substance use in young adulthood. ${ }^{15}$

\section{Cognitive-Behavioral Intervention for Trauma in Schools (CBITS). CBITS is a} cognitive-behavioral intervention provided by mental health clinicians, aimed at reducing trauma symptoms among at-risk elementary school children. The program uses a cognitive and behavioral approach to improve the student's adjustment to past traumatic events (violence, natural disasters) by teaching students relaxation techniques, social problem solving, and cognitive restructuring. The program is aimed at the general school population, but has flexible programming so that it can target relevant racial, ethnic, and socio-economic groups. Results from several trials demonstrate posttraumatic stress symptom reduction immediately post intervention and at 3- and 6-month follow-up. ${ }^{36-38}$ Effectiveness was most salient among violence exposed youth ${ }^{36}$ and youth exposure to natural disasters. ${ }^{37}$ Multiple respondents’ reports have helped to solidify consistency of symptom reduction from several perspectives and across contexts (e.g., behaviors at home vs. school). Intervention fidelity has been established ${ }^{36}$ and consistency of implementation assists maintenance of program efficacy over time, across studies, and in community (urban) settings. ${ }^{37,38}$ 
The specific areas included under the scope, scale, and dose of each program are summarized in Table 2 and then described in more detail in Table 3 and the text below.

\section{Scope}

The first aspect of scope considered is the degree to which interventions are preventionas compared to treatment-oriented. As shown in Table 3, all programs provide Tier 1 (universal) support to all students. PBIS, FRIENDS, and SFL also adapt the Tier 1 (universal) program to meet the needs of at-risk students (Tier 2 approach). CBITS targets only Tier 2 (provide sessions for youth at-risk for posttraumatic stress and internalizing/externalizing problems, respectively). Two programs, PBIS and MindMatters, do this by means of 'continuous support' the programs are integrated into the framework of the existing curriculum as opposed to outside of it in a separate intervention activity).

The scope of interventions can vary with regard to the domains of child functioning that are targeted. Interventions aimed at improving 'mental health' are the focus of this review as the development of mental health interventions are both supported and represented by a well-studied body of literature. There are, however, a number of closely related fields covered by these programs - variously labeled as ‘social/emotional learning’ ('SEL'), ‘well-being’, 'resilience’, or 'bullying'. As noted in Table 3, 5 out of 8 of the programs included contain elements of SEL, although the primary target of these programs is mental health. CBITS is the only program that is formulated on a Tier 2 framework for at-risk youth, and is the only program that contains no SEL components.

Interventions that are solely or primarily SEL, although widely disseminated, have been excluded from this review. For example, Lions Quest, one of the most widely disseminated programs, describes itself as a purely SEL program and it does not overtly target mental health as a stated outcome. While it has been implemented in 90 countries (many of them LMIC) and has 
demonstrated effectiveness in several RCTs, these studies have generally investigated the relationship between SEL and one or more of the following outcomes: positive behavior, drug and alcohol awareness, connection to school, and academic performance. While these behaviors in turn could have a direct impact on mental health outcomes, the studies to date do not make an explicit link to psychosocial functioning.

\section{Scale}

The next dimension considered is the scale of preventive mental health intervention programs for children. For the purposes of this review, four aspects of scale were considered: the total number of children reached to date, the number of children reached per annum, whether the program has been implemented at scale (national, provincial, or state), and whether it has been implemented in a LMIC. As noted earlier in this paper, our estimates of scale were based primarily on telephone interviews with the developers or evaluators of each program. Because of the different ways that large scale programs are rolled out these numbers can only be estimates, hence rounded numbers are presented.

As shown in Table 1, the programs (and the approximate number of students reached to date) covered by this review include: (1) Positive Behavioral Interventions and Supports (PBIS; 10,500,000), (2) FRIENDS (8,000,000); (3) Positive Action (PA; 5,000,000), (4) Promoting Alternative Thinking Strategies (PATHS; 2,000,000), (5) Skills for Life (SFL; in Spanish “Programa Habilidades para la Vida” or pHPV); 1,000,000; (6) MindMatters (300,000); (7) The Good Behavior Game (GBG; 200,000) and (8) Cognitive-Behavioral Intervention for Trauma in Schools (CBITS; 97,250).

In terms of the geographical meaning of scale (national, state/province, or school district) Table 1, column 6 summarizes this information for the programs that were reviewed. Three of 
the programs have reached national scale (FRIENDS and MindMatters in Australia, and SFL in Chile); FRIENDS has also been implemented on a state/province level in the United States. A number of programs have been implemented by whole school districts including PATHS, PA, CBITS, PBIS, MindMatters, and GBG.

FRIENDS is one of the most globally disseminated programs, implemented in more than a dozen countries. FRIENDS has been implemented on a national, state, and district scale in Australia since the early 1990s, with the first randomized control trial completed in $2001 .{ }^{10}$ It has recently become a national program, made available by the government of New Zealand, to all elementary schools (although the rate of actual participation is unknown). Additionally, for the past five years FRIENDS has been an official program of the province of Alberta, Canada. The program has been adapted for use in the $\mathrm{UK},{ }^{39}$ as well as in Brazil, Mexico, Ireland, Japan Taiwan, Finland, Hong Kong, and Sweden .

SFL is another program running on a national scale. It is offered by the Chilean department of education and implemented in all 15 of the country's regions and most of its large school districts. The program targets schools with high levels of poverty and other indicators of social risk. As of 2016, SFL was running in more than 2000 elementary schools where it reaches about 320,000 first through fourth graders each year. Related programs for middle and preschool aged children bring the number of students reached each year to about half a million.

Finally, MindMatters/KidsMatter is a program provided by the government of Australia. It has been operating since 1996 and has been a national program for the past decade, with revisions and expansions (including the merging of preschool, primary, and young adult versions with the secondary school version). MindMatters, an Australian initiative that promotes children's mental health in the school setting. MindMatters was originally designed for 
secondary school students and has recently been expanded to provide a version for primary schools (KidsMatter), preschools, and young adults. Some estimates have suggested that MindMatters is used, in some form, in up to half of all secondary schools in Australia and to a similar proportion of elementary schools, with an implementation plan to ensure its incorporation in all the new Zealand elementary schools.

Although none of the other six programs covered by this review have been implemented at the state/province or national level, several have been implemented in whole school districts and/or a large number of individual schools and these include some of the programs that have actually reached the most students. The three such programs (PBIS, PA, and PATHS) have been implemented with a total of about 17,000,000 children thus far. These three programs have been operating for an average of about 25 years, highlighting their feasibility. All three of these programs have very strong affiliations with universities and/or research institutes and have been evaluated in (as described earlier) more than 30 RCT's.

It is important to note that of the eight programs in the current review, three have been implemented to scale in LMIC. For example, the FRIENDS program has been implemented in more than 800 schools in Brazil as well as in several dozen schools in Mexico. The second program, SFL, was created by Chilean health and mental health professionals with educational colleagues in the late 1990's when Chile was classified as a LMIC (Chile was reclassified as a HIC in 2013), and so for the purpose of this review, SFL is categorized as a LMIC program based on its history. SFL remains a 'homegrown' program with no replications in other LMIC or HIC. The third program to reach large scale implementation in LMIC is the GBG. While most replication studies of the GBG have been completed in HIC such as the US, the Netherlands, 
Belgium, France, and the UK, the program has also been implemented, on a smaller scale, in Brazil.

\section{Dose}

The concept of dose, although very important, can be difficult to calculate across programs since the units of intervention vary widely. While the dose of most programs can be described in terms of the number of hours of intervention each student receives per day/week/year, two of the programs (PBIS and MindMatters) focus on providing the intervention through what they call 'continuous support' which means that the program is integrated into the framework of the existing school curriculum and/or environment, rather than added on as an a new protocol. Teachers are trained to follow program concepts/guideline throughout the time that students are in the school setting, as noted in Table 3.

SFL which has been operating on a national scale for more than a decade, ${ }^{13,34,40}$ is a good example of several of the key concepts of dose used in this paper. SFL aims to be a comprehensive preventive intervention for mental health by providing all three tiers of intervention: mental health promotional activities for all students, their parents, and teachers (Tier 1) in a given school, a 15 session intervention for students identified through an annual screening as having problems in first grade (Tier 2), and referral to clinicians outside of school for students who are found to have more serious mental health problems (Tier 3).

As shown in Table 3, the SFL intervention for Tier 1 involves a dose of 5 hours of selfcare for all teachers in participating schools with some schools also providing a similar 2 hour module for parents and an additional 5 hours for all students. For Tier 2, all students who screen positive are referred to a 10-session (1.5 hours each) cognitive-behavioral type group run by 
mental health professionals. Another component of the program is that parents of the students identified as being at risk receive three sessions and the student's teacher, two sessions. In total, the Tier 2 intervention provides about 19 hours of intervention per student. For Tier 3, all students who are referred by their teachers as having serious problems are evaluated, either at their school or at home, for a one hour by the mental health clinician who runs the school workshops. Students who are found to need additional intervention (e.g., treatment for depression or ADHD) are referred to clinicians in the local community.

The dose of CBITS can be readily calculated. Although it is the smallest of the programs reviewed here its impact has been demonstrated in a number of experimental studies. ${ }^{36,38,41}$ The program provides 10 group sessions, 1-3 individual sessions for each student, two psychoeducational sessions for parents, and one teacher session. Student sessions occur weekly and last a full class period. PATHS is another program where the dose can be calculated in a relatively straightforward manner. The core program consists of 36-52 class lessons depending on the child's age. At each level, PATHS is taught an average of 2-3 times per week in 20-30 min sessions and includes lesson objectives and scripts to encourage systematic implementation (although teachers have flexibility and may adapt a lesson structure based on individual group needs and teacher style). Of note, both Positive Action and PATHS entail comparatively large dosages and therefore achieving fidelity in program implementation may be more challenging, especially in other cultures where more extensive translation and adaptation would be required.

In contrast, the FRIENDS program involves a smaller dosage with 10 weekly sessions and 2 booster sessions ( 1 and 3 months post program completion). Each session is 75-90 minutes in length. Sessions have a specific sequence, structure, and topic (cumulative effect). For both Tier 1 and Tier 2, the dose is about 17.5 to 21 hours per student. 
PA (both a Tier 1 and Tier 2 program) uses a smaller, specific intervention approach with children typically receiving 140 fifteen minute sessions (35 hours) per year. The GBG operates on a similar model with children receiving 30 minute lessons daily throughout the 36 week school year (90 hours). The game is played each day as part of normal instruction. Both PA and CBG provide lessons that are implemented through the general school curriculum and focus on self-regulation, social skills, and mental health promotion.

Unlike the six programs reviewed above, the dose that each participating student receives in the remaining two programs (MindMatters and PBIS) is more difficult to calculate. We have classified these programs as providing the intervention through a systemic framework. For example, core elements of PBIS are integrated into the philosophy of the full school/organization, woven into traditional subject lessons rather than pulled out as distinct mental health lessons (see Table 3 for more detailed description) so that the interventions received by individual students is impossible to calculate. PBIS is provided by school staff and teachers with specific training in the model. MindMatters provides another example of the way that a continuous support/framework approach makes it difficult to assess individual student dose. Like PBIS, MindMatters provides structure and guidance within the school, but is malleable and thus allows organizations to build curriculum that fits the needs of the individual school, ${ }^{35}$ again making it difficult to compute the dose of the program received by individual students. MindMatters has components for different stakeholders in the school including pupils, principals, teaching and non-teaching staff, parents and the community around the school. Some of the manuals and exercises are directed to pupils, others are for school staff. Congruent with its universal approach, teachers gain skills to promote and support all students regardless of mental health status ${ }^{42}$. 


\section{Discussion}

This review has identified the school-based preventive interventions for children's mental health which have reached a large population, whether this is defined in terms of numbers of participating children or administrative entities. All of these programs have demonstrated their effectiveness using RCTs and/or quasi-experimental designs. Of note, the largest programs contain both Tier 1 and Tier 2 approaches, combining both universal prevention and targeted intervention for students most at-risk for psychosocial dysfunction, suggesting the benefit of multi-tiered and universal interventions, especially in school settings.

The quality of evidence regarding dissemination and experimentally proven efficacy for the school-based interventions identified in this review is moderate to strong. Findings from these studies indicate the implementation of the large-scale programs in several U.S. states as well as in Australia, and other HIC, and these programs are beginning to be implemented in LMICs, most notably India, Brazil, and Mexico.

With regard to implementation in LMIC, it is important to note that this review has found that the implementation of large scale school based mental health programs in LMIC has been confined only to programs that were created in LMIC or if they were created in HIC, after significant revisions (as with the FRIENDS program) to account for cultural heterogeneity. Despite this caveat, and the small number of programs, this review provides evidence that largescale school-based programs can be implemented in a variety of diverse cultures and preliminary evidence for significant, measureable positive effects on students' emotional, behavioral, and academic outcomes across a range of cultures, languages, and educational models. There is also growing evidence in LMIC as well as HIC that large scale programs are sustainable, with all of 
those reviewed here having operated for more than ten years (and in some cases two or three decades).

Programs vary widely on target behavior, tier, and dosage. However, the majority of the programs (7 out of 8) do contain at least a Tier 1 level intervention and are implemented on the district level. Six programs are implemented by a trained teacher in the classroom and two are embedded into the school curriculum through a system of continuous support. In these types of programs, children have easy access to a school-wide atmosphere of mental health promotion and this may contribute to the ability of these programs to reach millions of children and therefore grow to scale. Only two programs (SFL and CBITS) have a short-term directed intervention implemented by a mental health professional. Nonetheless, there is evidence that both these short-term targeted programs and the curriculum-based programs (e.g., PATHS) have long-term positive effects on behavior.

Programs that provide interventions to the same children over multiple years (e.g., GBG and PA) offer the possibility of cumulative and thus perhaps even greater impact on preventive and treatment outcomes. It is interesting to note that, of the programs reviewed here, it is only one of these (GBG) that has produced evidence that its positive effects persist over an extended period of time (for more than a decade, from elementary school into adulthood. ${ }^{18}$ Research that explores the factors associated with longer term and larger effects as well as studies exploring the conditions common to programs that have grown to scale are other important areas for future study.

Although the primary focus of this review is on the largest scale school-based mental health interventions, some types of mental health interventions for children that are not conducted at schools are worthy of note. For example, the US Head Start program can be viewed 
as the largest and longest running program for children's mental health in the world since it includes mental health as well as physical and dental health, nutrition, social-emotional development, and school readiness in its core areas of intervention. ${ }^{43}$ Now reaching more than 1 million pre-school children each year in the USA alone, as well as many more children through similar programs in other countries, it has probably provided at least some Tier 1 mental health services to over 30 million children since its inception in 1965. Head Start focuses on children from very low income families, and has features consistent with targeted mental health preventive interventions. ${ }^{43,44}$ The mixed, and at times controversial, findings from the many evaluations of Head Start should, however, sound a cautionary note of the complexity of evaluating mental health and all of the other types of social and emotional programs for children. ${ }^{45}$

A number of other well-known programs such as Incredible Years, ${ }^{46}$ Nurse Family Partnership, ${ }^{47}$ and Triple $\mathrm{P},{ }^{48}$ also attempt to promote child mental health for toddlers, infants, or prenatally with interventions aimed at mothers and are now also reaching large populations. It may also be important to note at this point that since our review was confined to studies published in English, it is possible that there are other large scale programs (both school- and non-school-based) that we are not aware of.

Even without including these programs, and still excluding the somewhat more generic social and emotional learning programs like Lion’s Quest that undoubtedly also enhance children's mental health, the school-based mental health programs discussed in this review appear to have reached more than 27 million children over the last decade and many of these programs have collected systematic data on outcomes. At a time of increasing data collection, the availability of large data sets from programs like these provides new opportunities to evaluate 
child mental health programs in multiple spheres, combining existing educational databases with those from mental health, health, and social service agencies, on a regional, state, or national scale. The availability of such data should facilitate and better capture the multiple effects of these interventions in the short, medium and long term by facilitating a mixed method approach to studying the effects of these often-complex interventions.

Even without more definitive evidence of impact, child mental health programs have grown, and probably will continue to increase in size and number and types of programs, leading to substantial numbers of children exposed to preventive mental health interventions. We must now focus on developing frameworks to match existing and new programs to the expressed needs of key stakeholders (such as schools- both students and staff- and community groups). With the growing availability and diversity of programs we can now pay greater attention to assessing the processes and practices of implementation that are associated with successful, widely disseminated, and sustainable programs, as well as to program limitations or liabilities that may impede adoption. Concepts like fidelity, appropriateness to local settings, penetration and adoption must be taken into account as we study what has and has not worked in the scaling up of large programs such as these. Finding ways to incorporate data from implementation and outcome measures and aligning them with the broader outcome interests of schools and their assessment procedures will maximize our ability to learn from these programs and disseminate and implement them further.

Given the call to improve the accessibility and relevance of services to school-aged populations, there is a need to focus on improving the evaluative frameworks of school based mental health programs over the coming years. Research to date supports the long held belief that such school-based programs continue to be one of the most promising types of preventive 
mental health interventions available for children. Better application of implementation science to the programs described in this review, have the potential to provide the foundation needed to develop the evidence-based and improve the health outcomes of the next generation.

Acknowledgements: The authors gratefully acknowledge the support of the Fuss Family Fund, and would like to thank Javier Guzmán for his comments on earlier drafts of this manuscript and Cara Lucke for her help preparing it for submission. 


\section{References}

1. Polanczyk GV. Addressing the Evidence Gap on Preventive Interventions. Journal of the American Academy of Child and Adolescent Psychiatry 2015;54:793.

2. Patton GC, Coffey C, Cappa C, et al. Health of the world's adolescents: a synthesis of internationally comparable data. Lancet 2012;379:1665-75.

3. Kieling C, Baker-Henningham H, Belfer M, et al. Child and adolescent mental health worldwide: evidence for action. The Lancet 2011;378:1515-25.

4. Patton GC, Sawyer SM, Santelli JS, et al. Our future: a Lancet commission on adolescent health and wellbeing. Lancet 2016.

5. Fazel M, Hoagwood K, Stephan S, Ford T. Mental health interventions in schools in high-income countries. The Lancet Psychiatry 2014;1:377-87.

6. Fazel M, Patel V, Thomas S, Tol W. Mental health interventions in schools in lowincome and middle-income countries. The Lancet Psychiatry 2014;1:388-98.

7. Polanczyk GV. Addressing the Evidence Gap on Preventive Interventions. J Am Acad Child Psy 2015;54:793.

8. Durlak JA, Weissberg RP, Dymnicki AB, Taylor RD, Schellinger KB. The impact of enhancing students' social and emotional learning: A meta analysis of school based universal interventions. Child dev 2011;82:405-32.

9. Bradshaw CP, Pas ET. A statewide scale up of positive behavioral interventions and supports: A description of the development of systems of support and analysis of adoption and implementation. School Psych Rev 2011;40:530.

10. Shortt AL, Barrett PM, Fox TL. Evaluating the FRIENDS program: A cognitivebehavioral group treatment for anxious children and their parents. J Clin Child Psychol 2001;30:525-35.

11. Flay BR, Allred CG. Long-term effects of the Positive Action ${ }^{\circledR}$ program. Am J Health Behav 2003;27:S6-S21.

12. Greenberg MT, Kusche CA, Cook ET, Quamma JP. Promoting emotional competence in school-aged children: The effects of the PATHS curriculum. Dev Psychopathol 1995;7:117-36.

13. Guzman MP, Jellinek M, George M, et al. Mental health matters in elementary school: First-grade screening predicts fourth grade achievement test scores. Eur Chile Adoles Psy 2011;20:401-11.

14. Wyn J, Cahill H, Holdsworth R, Rowling L, Carson S. MindMatters, a whole-school approach promoting mental health and wellbeing. Aust Nz J Psychiat 2000;34:594-601.

15. Kellam SG, Brown CH, Poduska JM, et al. Effects of a universal classroom behavior management program in first and second grades on young adult behavioral, psychiatric, and social outcomes. Drug Alcohol Depend 2008;95:S5-S28.

16. Jaycox LH, Kataoka SH, Stein BD, Langley AK, Wong M. Cognitive behavioral intervention for trauma in schools. Journal of Applied School Psychology 2012;28:23955.

17. Benedict EA, Horner RH, Squires JK. Assessment and implementation of positive behavior support in preschools. Top Early Child Spec 2007;27:174-92.)

18. Bradshaw CP, Mitchell MM, Leaf PJ. Examining the effects of schoolwide positive behavioral interventions and supports on student outcomes results from a randomized controlled effectiveness trial in elementary schools. J Posit Behav Interv 2010;12:133-48. 
19. Horner RH, Sugai G, Smolkowski K, et al. A randomized, wait-list controlled effectiveness trial assessing school-wide positive behavior support in elementary schools. J Posit Behav Interv 2009.

20. Horner RH, Sugai G, Smolkowski K, et al. A randomized, wait-list controlled effectiveness trial assessing school-wide positive behavior support in elementary schools. Journal of Positive Behavior Interventions 2009.

21. Bradshaw CP, Mitchell MM, Leaf PJ. Examining the effects of schoolwide positive behavioral interventions and supports on student outcomes results from a randomized controlled effectiveness trial in elementary schools. Journal of Positive Behavior Interventions 2010;12:133-48.

22. Childs KE, Kincaid D, George HP, Gage NA. The relationship between school-wide implementation of positive behavior intervention and supports and student discipline outcomes. Journal of Positive Behavior Interventions 2016;18:89-99.

23. Barrett PM, Sonderegger R, Xenos S. Using FRIENDS to combat anxiety and adjustment problems among young migrants to Australia: A national trial. Clinical Child Psychology and Psychiatry 2003;8:241-60.

24. Dadds MR, Spence SH, Holland DE, Barrett PM, Laurens KR. Prevention and early intervention for anxiety disorders: a controlled trial. Journal of Consulting and Clinical Psychology 1997;65:627.

25. Stallard P, Skryabina E, Taylor G, et al. Classroom-based cognitive behaviour therapy (FRIENDS): a cluster randomised controlled trial to Prevent Anxiety in Children through Education in Schools (PACES). The Lancet Psychiatry 2014;1:185-92.

26. Bernstein GA, Bernat DH, Victor AM, Layne AE. School-based interventions for anxious children: 3-, 6-, and 12-month follow-ups. Journal of the American Academy of Child \& Adolescent Psychiatry 2008;47:1039-47.

27. Li K-K, Washburn I, DuBois DL, et al. Effects of the Positive Action programme on problem behaviours in elementary school students: A matched-pair randomised control trial in Chicago. Psychology and Health 2011;26:187-204.

28. Flay BR, Allred CG. Long-term effects of the Positive Action ${ }^{\circledR}$ program. American Journal of Health Behavior 2003;27:S6-S21.

29. Flay BR, Allred CG, Ordway N. Effects of the Positive Action program on achievement and discipline: Two matched-control comparisons. Prevention Science 2001;2:71-89.

30. Domitrovich C, Durlak J, Goren P, Weissberg R. Effective social and emotional learning programs: Preschool and elementary school edition. 2013 CASEL guide. In; 2013.

31. Crean HF, Johnson DB. Promoting alternative thinking strategies (PATHS) and elementary school aged children's aggression: Results from a cluster randomized trial. Am J Commun Psychol 2013;52:56-72.

32. Humphrey N, Barlow A, Wigelsworth M, et al. A cluster randomized controlled trial of the Promoting Alternative Thinking Strategies (PATHS) curriculum. Journal of School Psychology 2016;58:73-89.

33. Schonfeld DJ, Adams RE, Fredstrom BK, et al. Cluster-Randomized Trial Demonstrating Impact on Academic Achievement of Elementary Social-Emotional Learning. School Psychol Quart 2014;30:406.

34. Guzmán J, Kessler RC, Squicciarini AM, et al. Evidence for the effectiveness of a national school-based mental health program in Chile. J Am Acad Child Psy 2015;54:799-807. e1. 
35. Rowling L. School mental health promotion: MindMatters as an example of mental health reform. Health Promot J Austr 2007;18:229-35.

36. Stein BD, Jaycox LH, Kataoka SH, et al. A mental health intervention for schoolchildren exposed to violence: a randomized controlled trial. JAMA 2003;290:603-11.

37. Jaycox LH, Cohen JA, Mannarino AP, et al. Children's mental health care following Hurricane Katrina: A field trial of trauma focused psychotherapies. J Trauma Stress 2010;23:223-31.

38. Kataoka SH, Stein BD, Jaycox LH, et al. A school-based mental health program for traumatized Latino immigrant children. J Am Acad Child Psy 2003;42:311-8.

39. Stallard P. Mental health prevention in UK classrooms: The FRIENDS anxiety prevention programme. Emot Behav Diffic 2010;15:23-35.

40. Murphy JM, Guzmán J, McCarthy AE, et al. Mental health predicts better academic outcomes: a longitudinal study of elementary school students in Chile. Child Psychiat Hum D 2014;46:245-56.

41. Jaycox LH, Cohen JA, Mannarino AP, et al. Children's mental health care following Hurricane Katrina: A field trial of trauma focused psychotherapies. J Trauma Stress 2010;23:223-31.

42. Askell-Williams H, Lawson MJ, Murray-Harvey R. Teaching and learning about mental illnesses: An Australian perspective. Int J Ment Health Promot 2007;9:26-36.

43. Jellinek MS, Bishop-Josef SJ, Murphy M, Zigler EF. Mental health in Head Start: Leave no child behind. NHSA Dialog: A Research-to-Practice Journal for the Early Intervention Field 2005;8:25-35.

44. Vogel CA, Boller K, Xue Y, et al. Learning As We Go: A First Snapshot of Early Head Start Programs, Staff, Families, and Children: Mathematica Policy Research; 2011.

45. Elango S, García JL, Heckman JJ, Hojman A. Early childhood education. Bonn, Germany: National Bureau of Economic Research; 2015.

46. White $\mathrm{C}$, Webster-Stratton $\mathrm{C}$. The incredible years baby and toddler parent programmes: Promoting attachment and infants' brain development. International Journal of Birth and Parent Education 2014;2:31-5.

47. Eckenrode J, Campa M, Luckey DW, et al. Long-term effects of prenatal and infancy nurse home visitation on the life course of youths: 19-year follow-up of a randomized trial. Arch Pediat Adol Med 2010;164:9-15.

48. Prinz RJ, Sanders MR, Shapiro CJ, Whitaker DJ, Lutzker JR. Population-based prevention of child maltreatment: The US Triple P system population trial. Prev Sci 2009;10:1-12. 